\title{
Conhecimento e aplicabilidade dos métodos não farmacológicos utilizados pelos enfermeiros obstetras para alívio da dor no trabalho de parto
}

\begin{abstract}
RESUMO|O processo de parturição é um evento fisiológico, que ocasiona contrações uterinas e dilatação cervical, o feto exerce pressão sobre as estruturas do assoalho pélvico materno aumentando a intensidade da dor. Este estudo buscou evidenciar o conhecimento e aplicabilidade dos métodos não farmacológicos utilizados pelos enfermeiros obstetras para alívio da dor na parturição. Trata-se de uma pesquisa de campo, com abordagem qualitativa, onde foram entrevistados 20 enfermeiros obstetras numa maternidade de referência Materno Infantil no Estado do Pará no período de outubro a novembro de 2016. Evidenciou-se o conhecimento dos enfermeiros obstetras sobre os métodos não farmacológicos, entretanto, somente uma pequena parcela dos profissionais utilizam os métodos em benefício da parturiente, devido a carga de trabalho ou falta de estrutura. Mesmo com as dificuldades encontradas nas práxis do cuidado os enfermeiros buscam vencer os obstáculos que aparecem no exercício profissional.
\end{abstract}

Palavras-chaves: Enfermagem Obstétrica, Parturição, Dor.

ABSTRACT I The process of parturition is a physiological event, which causes uterine contractions and cervical dilatation, the fetus exerts pressure on the structures of the maternal pelvic floor, increasing the intensity of the pain. This study aimed to demonstrate the knowledge and applicability of nonpharmacological methods used by obstetrical nurses to relieve parturition pain. This is a field research with a qualitative approach, where 20 obstetrician nurses were interviewed in a Maternal Infant Reference Maternity Hospital in the State of Pará from October to November 2016. The obstetrical nurses' knowledge about methods However, only a small proportion of these professionals use these methods for the benefit of the parturient, due to work load or lack of structure. Even with the difficulties found in the practice of care nurses seek to overcome the obstacles that appear in the professional practice.

Keywords: Obstetric Nursing, Parturition, Pain.

RESUMEN | El proceso de parturión es un evento fisiológico, que ocasiona contracciones uterinas y dilatación cervical, el feto ejerce presión sobre las estructuras del suelo pélvico materno aumentando la intensidad del dolor. Este estudio buscó evidenciar el conocimiento y aplicabilidad de los métodos no farmacológicos utilizados por los enfermeros obstetras para alivio del dolor en la parturición. Se trata de una investigación de campo, con abordaje cualitativo, donde fueron entrevistados 20 enfermeros obstetras en una maternidad de referencia Materno Infantil en el Estado de Pará en el período de octubre a noviembre de 2016. Se evidenció el conocimiento de los enfermeros obstetras acerca de métodos no farmacológicos, sin embargo, sólo una pequeña parte de estos profesionales utilizan tales métodos en beneficio de la parturienta, debido a la carga de trabajo o falta de estructura. Incluso con las dificultades encontradas en las práxis del cuidado los enfermeros buscan vencer los obstáculos que aparecen en el ejercicio profesional.

Palabras claves: Enfermería Obstétrica, Parturición, Dolor.

\section{Elyade Nelly Pires Rocha Camacho}

Enfermeira Obstetra, Mestre em Enfermagem, Doutoranda em Doenças Tropicais - Universidade Federal do Pará.

\section{Wanderson Luís Teixeira}

Bacharel em enfermagem pela Faculdade Pan Amazônica - FAPAN. Pós-graduando em Ginecologia e Obstetrícia, pela Escola Superior da Amazonia - Esamaz.

\section{Ana Carolina Gusmão}

Bacharel em enfermagem pela Faculdade Pan Amazônica - FAPAN. Especialista em Ginecologia e Obstetrícia, pela Escola Superior da Amazonia - Esamaz. PósGraduanda em Pediatria e Neonatologia, pela Escola Superior da Amazonia - Esamaz.

\section{Luciana Ferreira do Carmo}

Bacharel em enfermagem pela Faculdade Pan Amazônica-FAPAN, Belém-PA.

\section{Rosiane Luz Cavalcante}

Bacharel em enfermagem pela Faculdade Pan Amazônica - FAPAN, Belém-PA.

\section{Elisa Feitosa da Silva}

Doutora em Filosofia da Enfermagem pela Universidade Federal de Santa Catarina, Belém-PA.

Recebido em: 26/05/2019 Aprovado em: 28/08/2019
INTRODUÇãO

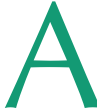

maternidade é um marco de grande significado pelas muIheres e representa o início de um novo ciclo, consagrando de maneira concreta o papel feminino, apesar de a grande maioria associar esse momento à forte dor e ao sofrimento ${ }^{(1)}$.

Apesar de ser considerado um evento fisiológico, o trabalho de parto e parto é envolto por modificações mecânicas e hormonais, que ocasiona contrações uterinas resultando na dilatação cervical e na descida da apresentação fetal, tanto na fase de dilatação quanto na fase de descida, a dor se apresenta de maneira intensa por vezes contínua e difusa ${ }^{(1)}$.

Contudo a dor não pode ser associada unicamente ao processo fisiológico, 
pois, outros fatores contribuem, como o medo, o estresse e a tensão, é evidente a importância da dor, e que a mesma deve ser minimizada a fim de evitar prejuízos ao binômio mãe e filho, onde os métodos não farmacológicos é uma alternativa que proporciona o alívio da dor na evolução do trabalho de parto (TP) ${ }^{(1,2)}$.

A Organização Mundial de Saúde (OMS) desenvolveu diversas pesquisas voltadas ao estímulo do parto normal, preconizando o evento, parto com o mínimo de intervenções, isto é, somente em caso de insegurança relacionado à saúde da mãe e criança. No entanto uma razão necessária para interferir sobre o processo fisiológico ${ }^{(1)}$. De acordo com a Diretriz Nacional de Assistência ao Parto Normal, é de livre escolha da parturiente os recursos não farmacológicos para alívio da dor durante o trabalho de parto, isto que as opções são diversas, entre as comprovadas cientificamente ${ }^{(2)}$.

Neste contexto a humanização da assistência em saúde às parturientes, surge como uma alternativa a transformação do cenário existente no domínio do parto fisiológico, que necessita de grandes mudanças nos diversos estágios que o compõem, a arquétipo das supostas dificuldades do não uso de recursos não farmacológicos no trabalho de parto e da falta de qualidade nos serviços de saúde ${ }^{(3)}$.

Considera-se fulcral ressaltar e aquilatar os dizeres da mulher no trabalho de parto visto que este processo é enfrentado de forma peculiar por cada paciente. O uso dos métodos não farmacológicos tem apresentado importante destaque e novos estudos vêm evidenciando a sua utilização como estratégia de alívio da dor ${ }^{(4)}$. Sendo assim, novas pesquisas que envolvam os métodos não farmacológicos são essenciais para dar o suporte clínico durante o trabalho de parto, assim como estudos que focalizem a preferência das parturientes nessas táticas, imprescindível para expansão da humanização e a qualidade na assistência ao parto.

A importância deste estudo caracteriza-se em virtude das recentes políticas que incentivam novas abordagens na assistência prestada pela enfermagem às parturientes. Frente a este contexto, emergiu a seguinte questão, a fim de nortear este estudo: Qual a compreensão do enfermeiro(a) obstetra sobre aplicabilidade dos métodos não farmacológicos período de parturição? A partir da resolução desta questão, pretende-se evidenciar o conhecimento e aplicabilidade dos métodos não farmacológicos, utilizados pelos enfermeiros. Tendo como objetivo evidenciar o conhecimento e aplicabilidade dos métodos não farmacológicos utilizados pelos enfermeiros obstetras para alívio da dor no trabalho de parto.

\section{MÉTODOS}

Tratou-se de uma pesquisa de campo, do tipo exploratório, com abordagem qualitativa e descritiva. A pesquisa foi realizada na Fundação Santa Casa de Misericórdia (FSCMPA), hospital de referência em atendimento de saúde Materna e Neonatal do estado do Pará. Realizou-se entrevistas com enfermeiros obstetras que atuam ou estão alocados na triagem obstétrica, nas salas de pré-parto, parto e puerpério ( $P P P$ 's). A pesquisa foi realizada nos meses de outubro a novembro de 2016. Utilizou-se como critério de inclusão enfermeiros especialistas em obstétrica e ativos na referida maternidade com no mínimo 6 meses de atuação, no qual gerou uma amostra de 20 entrevistados aptos. Foram excluídos 8 enfermeiros, por não possuírem vínculo institucional e enfermeiros em licença e/ou período de férias. Para a análise do material coletado nas entrevistas utilizou-se o método Bardin, seguindo sistematicamente as fases de pré-análise, exploração do material e tratamento dos resultados obtidos e interpretação. $\mathrm{Na}$ fase interpretativa, inicialmente, as entrevistas foram transcritas e realizadas repetidas leituras atentas aos discursos, e, na sequência, caracterizou-se em quatro eixos temáticos. A etapa consistiu no exercício interpretativo pretendido após a finalização da análise dos dados. O estudo foi aprovado pelo Comitê de Ética em Pesquisa da instituição da FSCMPA, em 06/10/2016, sob o número de parecer 1.763.964. CAAE: 59544416.3.0000.5171, sendo consideradas todas as exigências contidas na resolução 466/12, que rege as pesquisas com seres humanos.

\section{RESULTADOS}

1. Métodos Não Farmacológicos Conhecimento e aplicabilidade

Nesta categoria de análise, os 20 enfermeiros entrevistados disseram conhecer os métodos não farmacológicos para o alívio da dor no trabalho de parto. Porém 17 afirmaram que apesar de conhecer os métodos, encontram dificuldades na práxis devido falta de recursos, estruturas e sobrecarga de trabalho.

Os métodos não farmacológicos mais citados neste estudo foram: massagem lombossacral, exercícios respiratórios, banho de imersão citados por todos os entrevistados, seguidos do uso da bola suíça, deambulação, movimentos pélvicos (cavalinho), musicoterapia, e aromaterapia.

\subsection{MASSAGEM LOMBOSSACRAL}

Nessa pesquisa com enfermeiros obstetras quando perguntados sobre os benefícios da massagem lombossacral, eles demonstraram conhecimento sobre a técnica, conforme evidencias dos relatos transcritos a seguir:

“...Massagem lombossacral é um método de relaxamento importantíssimo para o alívio da dor, pois ao promover relaxamento por meio do toque conseguimos diminuir o estresse, fazendo com que essa mulher libere mais ocitocina, também conseguimos que o fluxo sanguíneo aumente possibilitando melhor oxigenação dos tecidos" (Sálvia)

“...A massagem lombossacral é um procedimento que pode ser 
realizado tanto pelo profissional que está assistindo o parto, tanto pelo acompanhante, pois trata-se de um método simples em que se coloca a mão espalmada sobre fundo uterino da mulher e a outra mão, também espalmada, sobre a região lombossacral, onde é realizado movimentos circulares, até o término de cada contração uterina" (Rósea)

\subsection{EXERCÍCIOS RESPIRATÓRIOS}

Em relação aos benefícios dos exercícios respiratórios, os enfermeiros obstetras referiram:

“...Quando se proporciona a parturiente uma respiração adequada durante as contrações, e também no Intervalo entre elas, há um relaxamento que diminui a ansiedade que permeia o trabalho de parto" (Caqui).

"Os exercícios respiratórios no trabalho de parto ajudam bastante a controlar o estresse que é vivido pela mulher e pelos familiares no momento do parto, e a técnica ajuda bastante principalmente no período expulsivo, onde há uma diminuição de oxigênio para o bebê devido a intensidade e a frequência das contrações" ( Bronze).

Ainda referente a respiração, o fato de olhar nos olhos da parturiente, lembrá-la de respirar sempre que ela estiver saindo do seu centro e perdendo a confiança na sua capacidade de vivenciar o trabalho de parto, também foram encontradas nas falas dos entrevistados:

“...Quando percebemos que a mulher esta insegura, procuramos fazer os exercícios respiratório, respirando junto com elas, instigando a confiança da paciente no profissional"(Amarelo).

"...A gente faz a mulher respirar de forma que a inspiração seja mais curta que a expiração, fazendo ela soltar o ar bem devagarzinho"(Lilás).

\subsection{BANHO AQUECIDO}

Neste estudo todos os enfermeiros entrevistados descreveram algum benefício relacionado ao banho de imersão, expuseram ainda que por não possuir estrutura física no hospital, eles gostariam de incentivar o banho de chuveiro com água aquecida como método de alívio da dor, no entanto, apenas em poucas situações conseguem realizar de maneira adequada. Neste tópico, os enfermeiros obstetras, entrevistados relataram que a água morna induz a vasodilatação, o que promove relaxamento muscular. Conforme relata o enfermeiro Hortelã:

“...Quando fazemos o uso do mecanismo de alívio da dor por este método proporcionamos a redução da liberação de catecolaminas e elevação das endorfinas, e assim conseguimos a redução da ansiedade e promovemos a satisfação da parturiente" (Hortelã).

Relacionando o banho e a fase da dilatação o enfermeiro (Rósea) descreve da seguinte maneira:

“...geralmente não tem diferença quanto a evolução da dilatação, apenas de conforto."

\subsection{BOLA SUÍÇA}

Os enfermeiros entrevistados elucidaram que a bola suíça auxilia no trabalho de parto de forma veemente, especialmente durante o processo de descida e rotação do feto, sem contar que se trata de uma técnica lúdica, que diminui a sensação de dor, como relatam os entrevistados:

"...Me identifico como esse método, pois ajuda a mulher a diminuir sua dor, e facilita a descida do feto na pelve materna" (Cinza Outono)
“...Os benefícios são muitos para a mulher como: o fortalecimento da musculatura, o relaxamento e etc." (Esmeralda)

\subsection{DEAMBULAÇÃO}

Outro método destacado nos resultados é à deambulação, contudo, nessa pesquisa a técnica foi pouco usada pelos enfermeiros obstetras entrevistados, devido a infusão de ocitocina, que ainda dificulta o procedimento devido a punção venosa, pois traz incômodo e diminui a mobilidade da parturiente, proporcionando, o aumento das contrações uterinas o que é descrito nas falas dos entrevistados: "... caminhar durante o trabaIho de parto é uma técnica utilizada para promover relaxamento e aliviar a dor durante este período, mas aqui na maternidade é complicado pois a ocitocina é utilizada como rotina então mulher fica com dificuldades na mobilidade devido ao acesso venoso, além do que, depois que é instalada as contrações aumentam a intensidade em um período curto de tempo, fato que as restringe no leito" (Laranja).

\subsection{CAVALINHO}

Dentre os métodos não farmacológicos comumente utilizados pelos enfermeiros entrevistados, está o uso do cavalinho, este método segundo relato abaixo, promove o relaxamento e reduz a percepção dolorosa durante as contrações uterinas.

"...O cavalinho é muito aceito como forma terapêutica para o alívio da dor. Quando assisto meus partos, esse método me ajuda muito no controle das dores que as muIheres sentem" (Bronze lenda).

1.7 LIMITAÇÕES NO USO DE METODOS NÃO-FARMACOLÓGICOS 
A dor é uma experiência subjetiva, sua intensidade é diferente entre os indivíduos que a sentem, neste contexto faz-se necessário um olhar holístico para mulher no processo de parturição, visto que esta vivência é única e distinta, o que deve conduzir ao respeito, possibilitando os profissionais que tomem uma conduta integrativa e humanizada ${ }^{(4)}$.

Neste estudo também foram citados, dentre os entrevistados, outros métodos terapêuticos para o alívio da dor no momento da parturição tais como: a aromaterapia e musicoterapia, banho de Imersão, métodos não utilizados devido à falta de material ou questões estruturais, sendo perceptível na fala a seguir:

“...Há um tempo atrás, tinha uns acadêmicos que traziam uns óleos, incensos etc,que realmente proporcionavam melhora do bem estar das pacientes" (Azul).

“...O banho de imersão era realizado quando as banheiras prestavam, hoje fica difícil, só de asperção mesmo e olhe lá" (Verde).

\section{DISCUSSÃO}

Segundo as recomendações da Organização Mundial da Saúde, para o atendimento ao parto normal é falado que existem condutas úteis no trabalho de parto e que essas condutas deveriam ser encorajadas, pois são estratégias utilizadas para aumentar a tolerância à dor ${ }^{(2)}$.

O trabalho de parto e o próprio ato de parir perdeu seu significado com os avanços tecnológicos na medicina. Porém o mesmo precisa ser resignificado e visto como processo natural onde a finalidade é um binômio sem intervenções e saudável ${ }^{(6)}$. A dor é uma experiência subjetiva, sua intensidade é diferente entre os indivíduos que a sentem, neste contexto faz-se necessário um olhar holístico para mulher no processo de parturição, visto que esta vivência é única e distinta, o que deve conduzir ao respeito, possibilitando os profissionais que tomem uma conduta integrativa e humanizada ${ }^{(4)}$.

Nessa pesquisa quando indagando, os enfermeiros demonstraram conhecimento sobre a técnica e os benefícios da massagem lombossacral, corroborando com Reginaldo e colaboradores5, no qual descreve que houve uma redução significativa da dor na percepção das mulheres que realizaram a massagem lombossacral, evidenciando que as parturientes tiveram redução da dor pela terapêutica, em especial na fase latente do trabalho de parto.

Segundo Chan e colaboradores ${ }^{(7)}$, em sua pesquisa com 60 parturientes verificaram uma significativa influência no alivio da dor quando utilizar a massagem lombossacral. Ou seja, destacaram a estratégia como um valor terapêutico expressivo, capaz de promover conforto, relaxamento e aliviando a dor durante o trabalho de parto $^{(7)}$.

O processo de dor no trabalho de parto é oriundo da própria natureza humana, esta dor não se associa a patologias, mas na incrível experiência de gerar outras vidas ${ }^{(5)}$. De forma a oferecer uma assistência humanizada e de qualidade o enfermeiro obstetra juntamente com a equipe de enfermagem deve avaliar o que a mulher sabe sobre estratégias de alívio da dor e oferecer informações intrínsecas para encontrar quais abordagens são mais aceitáveis para a mulher, de maneira que a assistência humanizada propicie uma mudança de comportamentos para amenizar o sofrimento da mulher no processo fisiológico que é parto ${ }^{(1)}$.

Sendo assim, a massagem lombossacral sempre foi um dos meios mais naturais e instintivos de aliviar a dor e o desconforto no processo de parturição, uma vez que reduz a ansiedade e o estresse e concomitantemente promove o relaxamento muscular e diminui a fadiga muscular, que tem ação sedativa e analgésica, traz aumento da consciência corporal e produz benefícios emocionais de equilíbrio entre sistema simpático e parassimpático Embora, não exista um consenso relacionando a riscos e benefícios da massagem durante o trabalho de parto, na prática, observa-se que essa técnica tranquiliza a parturiente, aliviando a dor e a ansiedade, e conduzindo de maneira satisfatória o processo de parir ${ }^{(15)}$.

Contudo, muito embora que este método e sua aplicabilidade tornam a processo de parturição mais ameno do ponto de vista da sensação dolorosa, os enfermeiros envolvidos na assistência são limitados na execução da técnica, haja vista, que partejar requer tempo, e as atividades burocráticas dificultam a realização práticas de muitos profissionais envolvidas na assistência obstétrica.

No que diz respeito aos exercícios respiratórios no processo da parturição, este promove o relaxamento, diminui riscos de trauma perineal no momento expulsivo e 
melhora a oxigenação sanguínea da mãe e do produto da concepção(8).

Segundo os relatos dos enfermeiros foi percebido que os exercícios respiratórios, reduzem a sensação dolorosa e meIhora sobremaneira os níveis de saturação sanguínea de oxigênio, pois proporciona o relaxamento e diminui a ansiedade. Neste sentindo os exercícios respiratórios podem não ser suficientes na redução da sensação dolorosa durante o primeiro estágio do trabalho de parto, porém são eficazes na redução da ansiedade.

Corroborando com as falas dos enfermeiros, a respiração tem importância fundamental durante o trabalho de parto e no parto, porque promove o relaxamento, promove uma melhor concentração de todo o processo que esta vivenciando durante o trabalho de parto, diminui os riscos de trauma perineal no momento expulsivo, melhorando assim a oxigenação sanguínea da mãe e do feto ${ }^{(8)}$.

Por isso a parturiente deve inspirar pelo nariz e expirar pela boca durante as contrações uterinas, de maneira atenta, pausada e de forma consciente. É importante que o profissional acompanhe-a, respirando da mesma forma simultaneamente $^{(8)}$. Olhar nos olhos da parturiente, lembrá-la de respirar sempre que ela estiver saindo do seu centro e perdendo a confiança na sua capacidade de vivenciar o trabalho de parto $^{(9)}$.

A respiração orientada descritas nas falas dos enfermeiros, aplicada, nas atividades da assistência ao parto tem demonstrado um benefício para o alivio da dor durante o parto, melhora da oxigenação, contribuindo para desfechos com êxito para o binômio mãe e filho, isto precisa ser incentivado.

Nesta pesquisa os enfermeiros entrevistados descreveram alguns benefícios relacionado ao banho de imersão. A água aquecida induz a vasodilatação periférica e redistribuição do fluxo sanguíneo, promovendo relaxamento muscular ${ }^{(8)}$. O mecanismo de alívio da dor por este método é a redução da liberação de catecolaminas e elevação das endorfinas, reduzindo a ansiedade e promovendo a satisfação da parturiente. Muitas dificuldades foram encontradas na realização dessa técnica relatadas pelos enfermeiros, haja vista que a estrutura hospitalocentrica não dispõe de recursos suficientes como: banheiras, hidromassagem e "piscinas de parto". Apesar da existência de poucos estudos utilizando o banho de chuveiro durante o trabalho de parto, este recurso parece exercer influência positiva sobre a dor. Nos últimos anos, a imersão em água durante o trabalho de parto e o parto despertou interesse em muitos países, em resposta às solicitações femininas dessa forma de conforto para o alivio da sensação dolorosa ${ }^{(8)}$.

Em estudo sobre os efeitos do banho de imersão, Flora e seus colaboradores ${ }^{(10)}$ perceberam que as parturientes sobre o efeito do método possuíam no mínimo duas, e no máximo cinco contrações a cada 10 minutos. Enquanto as que não eram submetidas ao banho de imersão possuíam com a assistência do banho de imersão possuíam no mínimo uma e no máximo cinco contrações em menor tempo, e a maior frequência. Em relação a dilatação cervical, o mesmo autor assegurou, que não houve diferença significativa entre as parturientes do grupo experimental (uso do banho), e as do grupo de controle (não fizeram uso do banho).

Quando entrevistados sobre a utilização da bola suíça, foi relatado os benéficos oriundos desse método, pois a bola favorece um conforto significativo do assoalho pélvico da gestante. É uma técnica utilizada durante o trabalho de parto que auxilia na descida e rotação do concepto, pois trabalha a musculatura pélvica e oferece a mulher maior conforto físico e psicológico no ato de pariri ${ }^{(9,10)}$.

A parturiente pode ainda, fazer movimentos rotativos (de bambolê). Neste sentido a movimentação do quadril facilita a rotação do bebê, auxiliando-o a se deslocar para a posição correta. Sempre é recomendável que a parturiente segure as mãos do profissional de saúde ou do companheiro, para ficar com mais firme- za. Segundo os enfermeiros a bola Suíça é um recurso que deve ser utilizado com o objetivo de facilitar a adoção de postura vertical pela parturiente de forma confortável, um método simples e de fácil a acesso no processo de parturição ${ }^{(9)}$.

Apesar de ser um método efetivo e de baixo custo ainda faltam estudos para esclarecimentos sobre a prática a fim de que a parturiente se sinta segura ao utilizá-la. É importante salientar que a assepsia da superfície e para a prevenção da contaminação cruzada. Por exemplo, mais pesquisas com a realização de mais estudos e de que demonstrem qual o momento adequado para a utilização desse recurso, na hora do parto ${ }^{(11)}$.

Outro método destacado dessa pesquisa foi à deambulação, por ser vantajosa devido a ação gravitacional que auxilia a descida do feto, e outra vantagem da deambulação é que durante a movimentação, e na ocorrência das contrações, tendem a ficar menos dolorosas e ter mais efetividade ${ }^{(7)}$.

No estudo de Isamara e colaboradores12, com oitenta primigestas em trabalho de parto espontâneo, 54 fizeram uso de ocitócito e percorreram 1.696,22 metros, no entanto, 49 parturientes que completaram a dilatação cervical tiveram um tempo médio de 8,31 horas. Das que não fizeram uso dessa medicação, 26 parturientes percorreram uma distância média de 1.532,49 metros, durante a fase ativa do trabalho de parto, e completaram esta fase em 6,46 horas.

Contudo, nessa pesquisa a técnica foi pouco usada pelos enfermeiros obstetras Quando falamos da deambulação no processo de parir, percebemos como um recurso terapêutico utilizado para reduzir a duração do trabalho de parto, beneficiando-se do efeito favorável da gravidade e da mobilidade pélvica que atuam na coordenação miometrial e aumentam a velocidade da dilatação cervical e descida fetal. Alguns estudos demonstram que a deambulação aumenta a tolerância à dor no trabalho de parto. Mesmo tendo consciência dos benefícios oriundos deste mé- 
todo, os enfermeiros entrevistados relatam a dificuldade da execução dessa técnica por conta da rotina hospitalar que os impedem de efetivar de forma satisfatória por conta do uso restrito de movimentação ${ }^{(3)}$.

Apesar de ser considerado como um método de extrema eficiência, a rotina influência de maneira significativa na assistência ao parto, sendo assim, restrito o uso da livre movimentação durante o parto.

Um dos métodos terapêuticos utilizados no alivio da dor é o "cavalinho", que segundo os enfermeiros, apresentam um significativo efeito sob o processo de inibição da sensação dolorosa, pois o "cavalinho" é um equipamento disponível no pré-parto, bancos cuja utilização visa o relaxamento, aumento da dilatação e a diminuição da dor. O "cavalinho" é semelhante a uma cadeira com assento invertido, onde a gestante apóia o tórax e os braços jogando o peso para frente e aliviando as costas. Durante as contrações, a parturiente também pode ficar nessa posição para receber massagem na lombar, com a finalidade de relaxar e aliviar a dor do trabalho de parto ${ }^{(3,13)}$.
Nesta perspectiva, os métodos não farmacológicos para alívio da dor no parto representam ser de suma importância, uma vez que esses métodos auxiliam no conforto e diminui a ansiedade, medo, aflição da parturiente e consequentemente reduz a dor, e a sua utilização representa um parto menos doloroso. Contudo, é importante frisar que mesmo tendo conhecimento destes métodos que são essenciais com benefícios consideráveis, muitas das vezes acabam sendo abandonados pois não existem ferramentas que possam contribuir para a sua utilização.

\section{CONCLUSÃO}

Neste estudo ficou evidenciado o conhecimento dos enfermeiros obstetras sobre os métodos não farmacológicos. Eles auxiliam no alívio da dor no processo de parturição, entretanto, somente uma pequena parcela dos profissionais utilizam os métodos em benefício da parturiente, devido à carga de trabalho ou falta de estrutura física.
As questões que se relacionaram com o excesso de trabalho e a estrutura física, ainda se apresentam nas instituições de saúde, mesmo com os avanços e frente aos avanços e conquistas que foram agregados ao longo dos anos a respeito das técnicas, que podem ser utilizadas a fim de minimizar a experiência dolorosa e favorecer um parto mais digno e respeitoso.

Mesmo com as dificuldades encontradas nas práxis do cuidado os enfermeiros lidam com os obstáculos que se apresentam no exercício profissional.

Com o advento técnico científico, as técnicas de manejo da dor durante o trabalho de parto se tornaram dinâmicas e aceitáveis no universo da saúde. Sendo possível identificar que os enfermeiros conseguem aplicar alguns dos métodos, humanizando o cenário do parto, evitando procedimentos desnecessários e tornando o trabalho de parto mais prazeroso a parturiente.

*Extraído do Trabalho de Conclusão de Curso apresentado a Faculdade de Enfermagem da Faculdade Pan-Amazônica.

\section{Referências}

1. Aragão, TH; Vieira, SS; Fernandes, ETS; Silva, GM. Trabalho de parto e os métodos não farmacológicos para alívio da dor: Revisão Integrativa. INTERNATIONAL NURSING CONGRESS Theme: Good practices of nursing representations In the construction of society. May 2017; 9-12.

2. Ministério da Saúde (BR). Secretaria de Ciência, Tecnologia e Insumos Estratégicos, Departamento de Gestão e Incorporação de Tecnologias em Saúde. Diretrizes nacionais de assistência ao parto normal. [Internet] Brasília: Ministério da Saúde; 2017. [Acesso 6 abr 2017]. Disponível em: http://bvsms.saude. gov.br/bvs/publicacoes/diretrizes_nacionais_assistencia_parto_normal.pdf 3. Ministério da Saúde (BR). Secretaria de Ciência, Tecnologia e Insumos Estratégicos. Diretriz Nacional de Assistência ao Parto Normal: relatório de recomendação [Internet]. Brasília (DF);2016. [Acesso 12 jan 2017]. Disponível em: http://conitec.gov.br/images/Consultas/ 2016/Relatorio_Diretriz-PartoNormal_CP.pdf.

4. Araujo, RCG; Rocha, ELCA; Silva, EC; França, AMB. Recursos não farmacológicos para o alívio da dor no trabalho de parto: revisão integrativa. Ciências Biológicas e de Saúde Unit. Aracaju; Maio 2017; v. 4; n. 1; p. 123-134.

5. Mafetoni, RR; Shimo, AKK. Métodos não farmacológicos para alívio da dor no trabalho de parto: revisão integrativa. REME • Rev Min Enferm. 2014 abr/ jun; 18(2): 505-512.

6. Cechin, PL. Reflexões sobre o resgate do parto natural na era da tecnologia. Rev. bras. enferm. [Internet]. 2002; [acesso:20.02.2019]; 55(4):444-448. Disponível em: http://www.scielo.br/scielo.php?scrip$\mathrm{t}=$ sci_arttext\&pid=S0034-71672002000400015\&lng=en. http://dx.doi. org/10.5935/0034-7167.20020095.

7. Chan, MY; Wang, SY; Chen, CH. Effects of message on pain and anxiety during labor: a randomized controlled trial in Taiwan. Journal Adv. Nurs. 2002. April. 38 (1): 63-73.

8. Davim, RMB; Torres, GV; Dantas, J. C. Efetividade de estratégias não farmacológicas no alívio da dor de parturientes no trabalho de parto. Rev.esc. enferm. USP, 2009; 43(2):438-45.

9. Gallo RBS, Santana LS, Marcolin AC, Ferreira CHJ, Duarte G, Quintana SM. Recursos não-farmacológicos no trabalho de parto: protocolo assistencial. Rev. Rene, São Paulo, abr./jun. 2011; v. 39, n. 4, p. 86-93.

10. Bottosso, RS.(organizador). Manual do processo de enfermagem e sua aplicação no Centro Cirúrgico e Centro Obstétrico. Universidade Federal de Mato Grosso. Hospital Universitário Júlio Müller. Cuiabá, Mato Grosso. Outubro/2006; $1^{\text {a }}$ edição. p. 01-81.

11. Flora, MBS; Sonia, MJVO. 0 efeito do banho de imersão na duração do trabalho de parto. Rev. da Esc. de Enferm. da USP, 2006. v. 40, n. 1, p. 57-63. 12. Rodrigues, AC; JESUS, JP; LIMA, JJ; MELO, VLS; IZIDORO, LCR. Práticas interativas não farmacológicas no trabalho de parto, Revista Científica FacMais, Jun,2018. Volume XIII, Número 2.

13. Mamede, FV; Almeida, AM; Nakano, AMS; Gomes, FA; Panobianco, MS. 0 efeito da deambulação na duração da faze ativa do trabalho de parto. Esc. Anna Nery 2007; 11(3): 466-471.

14. Sescato, AC; Souza, SRRK; Wall, ML. Cuidados não-farmacológicos para alívio da dor no trabalho de parto: orientações da equipe de enfermagem. Cogitare Enferm. 2008; 13(4):585-90.

15. Cassol EGM, Canfield JT, Morais EN. Desempenho motor e emocional de parturientes durante $02^{\circ}$ período do trabalho de parto. Rev Bras Fisioter 2001; 5(1):9-16. 